\title{
Older Asian immigrants' perceptions of a health-assistive smart home
}

\author{
${ }^{1}$ Roschelle L. Fritz PhD RN FAAN ${ }^{\mathrm{a}, *},{ }^{1}$ Connie Kim Yen Nguyen-Truong PhD RN \\ Alumnus PCCN ${ }^{\mathrm{a}}$, Jacqueline Leung JD MS CHW', Junghee Lee $\mathrm{PhD}^{\mathrm{c}}$, Christine Lau \\ MA CACDI ${ }^{d}$, Cang Le BS ${ }^{e}$, Jane Kim MEd ${ }^{\mathrm{e}}$, Keith Wong BA ${ }^{\mathrm{e}}$, Thai Hien Nguyen BSN \\ $\mathrm{RN}^{f}$, Tuong Vy Le MS BS ${ }^{f}$, Jennifer I. Nevers BSN RN ${ }^{g}$, Anthony M. Truong BS RPh \\ ${ }^{a}$ College of Nursing, Washington State University, Vancouver, Washington, USA; ${ }^{b}$ Oregon \\ State University, Corvallis, Oregon, USA; ' ${ }^{C}$ chool of Social Work, Portland State Univer- \\ sity, Portland, Oregon, USA; ${ }^{d}$ Asian Health \& Service Center, Portland, Oregon, USA; \\ ${ }^{e}$ Community \& Public Health Department, Asian Health \& Service Center, Portland, Or- \\ egon, USA; ${ }^{f}$ Asian Community, Portland, Oregon, USA; ${ }^{g}$ College of Nursing, Washington \\ State University, Spokane, Washington, USA; ${ }^{1}$ Roschelle L. Fritz and ${ }^{1}$ Connie K.Y. Nguy- \\ en-Truong are co-first authors as they have done an equal amount of the work. *Corre- \\ sponding author: shelly.fritz@wsu.edu
}

\begin{abstract}
Background: The needs of the growing aging population will soon surpass available resources. Health-assistive smart homes may be one solution for extending independence and facilitating early health interventions thereby lowering healthcare costs and conserving resources. Perceptions of usability and adoption may differ across populations.

Objective: To explore diverse older Asian immigrants' perceptions of adoption of healthassistive smart home monitoring (smart home) and culturally-specific expectations.

Methods: A community-engaged research approach was employed. Participants $(n=55$; age 55+) were recruited through an immigrant community health and social services center in the United States. Participants joined one of four language-based group discussions (Chinese Cantonese, Chinese Mandarin, Vietnamese, or Korean). Scientific group-level assessment, a participatory qualitative data collection, and analysis method, was conducted in real-time with participants. The main ideas were transcribed, verified with participants, and translated into English.

Results: Intra- and inter-group themes were identified. Inter-group themes included: concerns about safety, features and functionality, trusting technology, households and family, and access (cost, internet, knowledge, and literacy). Intra-group themes included: Cantonese (access, radiation, user friendly, installation, technology knowledge required), Mandarin (access, emergency concerns, user friendly, locus of control, technology knowledge required, language), Vietnamese (limited technical knowledge, smart home capacity, availability, access, safety, cost, my home), and Korean (limited technical knowledge, functionality, household makeup, privacy, threats, availability and cost, my culture).

Conclusions: Immigrants showed interest in adopting smart home technology for agingin-place. The impact on family engagement and community expectations when using a technology that monitors individuals' daily in-home routines and activities must be acknowledged.
\end{abstract}

Keywords: Smart homes, sensors, culture, older adults, minority health

\section{INTRODUCTION}

The world's population is aging. By 2050, it is estimated that $22 \%$ of the world's population will be age 60 or over and by 2020 this group will outnumber children younger than 5 years old (World Health Organization, 2018). Managing the care of older adults is a global humanitarian concern. The number of persons capable of caring for older adults is disproportionately low compared to global projections of the growth of the older adult population (National Institute on Aging, 2017; World Health Organization, 2018). Concomitantly, many older adults want to re- main in their home as long as possible (International Federation on Ageing, 2011) and they are open to using smart home technology if it can support health and independence without compromising their safety and quality of life (Courtney, Demiris, \& Hensel, 2007; Fritz, Corbett, Vandermause, \& Cook, 2016; Peek et al., 2016).

Many older adults already use technologies such as computers, email, FaceTime or video calling, and smartphones. A novel technology designed to support older adults' efforts to agein-place, the health-assistive smart home (smart 
home), is under development in many countries (i.e., United States, England, Japan, Netherlands, and more). Smart homes typically use an array of sensors (e.g., infrared motion, door use, light, temperature, and humidity) and machine learning (ML) to monitor older adults' daily activities and changes in behavior patterns that are clinically relevant (Alberdi, Weakley, Goenaga, \& Schmitter-Edgecombe, 2018; Sprint, Cook, Fritz, \& Schmitter-Edgecombe; Rantz et al., 2015). Smart homes may or may not use cameras and/or microphones (Ye, Stevenson, \& Dobson, 2016). [The smart home used for this study is under development at Washington State University and does not use cameras or microphones. For more information see casas.wsu.edu.] However, this technology has not been widely adopted by older adults or the broader healthcare community. Given that (a) the promise of smart homes is creating wide-spread interest among healthcare workers (Dzissah, Lee, Suzuki, Nakamura, \& Obi, 2019; Helal \& Bull, 2019; Hunter et al., 2019), (b) responses to, and interaction with, technology vary based on socio-cultural context (Chen \& Chan, 2014; Helal \& Bull, 2019; Hong, Zhou, Fang, 2017; Lundin \& Mäkitalo, 2017; Mara, Gill, Babacan, \& Donahoo, 2011), and (c) all persons have a right to culturally sensitive care (including technology-assisted care) (American Academy of Nursing, 2011), studies exploring the connection between culture and smart home adoption are of significant value to patients, their families, and the greater society. Thus, the potential for diffusion of this innovation across populations and cultures should be studied.

\section{Theoretical framework}

Rogers' (1976) Diffusion of Innovation Theory frames this work. Rogers theory explains how innovations are assumed by populations over time. Rogers proposes people fall within a Bell Curve spectrum of openness to adopt. Categories across the spectrum are Innovator, Early Adopter, Early Majority, Late Majority, and Laggards. Innovators comprise just $2.5 \%$ of the population and they are the earliest adopters within a population. Early Adopters are the next most-likely category of people to adopt an innovation (13.5\%) followed by an Early Majority (34\%), Late Majority (34\%), and Laggards (16\%) (Rogers, 2003). Innovation attributes and adoption rates are impacted by perceptions related to the relative advantage of using an innovation (e.g., smart home monitoring) over the status quo (e.g., no in-home monitoring), compatibility with lifestyle and existing values, the innovation's complexity (usability), the end-user's ability to try it without harm or significant commitment, and the end-user's ability to observe others using it. We chose Diffusion of Innovation Theory over other technology acceptance theories or models (e.g., Technology
Acceptance Model, Unified Theory of Acceptance and Use of Technology) because Roger's theory contains a clear cultural component and because it has been used to study the impact of culture on the adoption of a variety of innovations (Sahin, 2006; Rogers, 2004), positioning it in the technology adoption arena as a robust theory. We also prefer this theory because it uses the language of adoption versus acceptance. As nurses, we think the word adoption is less presumptive regarding whether patients could, or should, use a smart home, sensor-based monitoring technology to extend independence and improve health outcomes.

\section{Culture and technology}

An important component of understanding the broad applicability of smart homes is transferability across diverse racial, ethnic, and cultural groups. Researchers at several universities have examined older adults' perceptions of adopting smart home technology (e.g., Washington State University - United States, University of Washington - United States, University of Missouri - United States, Fontys University of Applied Sciences - Netherlands, Tsinghua University - People's Republic of China). However, in a search of the smart home literature across five databases (Google Scholar, PubMed, IEEE Xplore, $\mathrm{CINAHL}$, and PsychInfo) only three studies were identified that specifically focused on exploring a connection between culture and older adults' perceptions of smart homes designed to extend independence and manage health. Chung, Thompson, Joe, Hall, and Demiris (2017) used qualitative methods with focus groups $(n=21)$ to examine Korean and Korean Americans' perceptions of smart home acceptability. These researchers found both groups were interested in potentially using smart home technology as a way to extend independence and increase safety. Participants indicated they were interested in smart homes because they were increasingly living alone due to loosening familial piety as a result of immigration, the mobility of modern society, and because of a decreasing satisfaction with healthcare. Jeong, Salvendy, and Proctor (2010) also examined the cultural aspects of Korean and American perceptions of using smart homes for aging-in-place. Jeong et al. used a psychometric survey $(n=452)$ to explore factors associated with design and operation preferences between cultures. Differences in preferences by culture were found. The study also illuminated the critical role culture plays in older adults' desire to use smart homes (i.e., smart home varieties, appliances, and connectivity of the various components of the smart home). Fritz, Corbett, Vandermause, and Cook (2016) took a different approach to explore the culture and smart homes. Rather than defining culture as a component of 
racial identity, this team allowed older adult participants in the United States to self-identify their culture. Cultural identities varied greatly with participants identifying themselves as part of 'educated', or 'female socialite,' or 'Midwest farmer', or 'generational mature silent' (and more) cultures. In this qualitative descriptive study $(n=$ 21) only two participants were non-White; both identified as 'Asian.' Fritz et al. (2016) found that culture (self-defined and comprised of socially constructed values and behaviors) would influence the adoption of smart homes designed to assist with aging-in-place.

We conducted the current qualitative study to expand and diversify data beyond previous qualitative studies, which only included persons of Korean descent. We also wanted to assess openness to the smart home as a precursor to deploying them to diverse older adult Asian immigrants. [We are working towards increasing data derived from persons of color for training our machine learning algorithms.] In this study, we expand on technology acceptance research that has focused solely on Koreans. We include a more diverse group of Asians to move understandings forward and prepare for conducting studies that increasingly include persons of color. Fifty-five older adult immigrants of Asian descent from four language groups and three countries of origin participated (China - various regions and dialects, Vietnam - three regions, and South Korea). This community-engaged qualitative descriptive study aimed to explore (a) older diverse Asian immigrants' knowledge and perceptions of smart homes, and (b) the influence of culture on adopting smart home monitoring. This study was determined to be exempt by the Washington State University Institutional Review Board.

\section{Methods}

We used community-engaged research (CEnR) approach to conduct this study. An academiccommunity partnership was created between Asian Health \& Service Center (AHSC) and Washington State University College of Nursing in the Pacific Northwest region of the United States. AHSC is a community-based health and social services center primarily serving a fast-growing Asian immigrant population in the Portland, Oregon metropolitan area. It is a trusted source of information within the local Asian immigrant community (Nguyen-Truong et al., 2017). The majority of constituents are Chinese, Vietnamese, and Korean immigrants with four main language groups: Chinese Cantonese (CC), Chinese Mandarin $(\mathrm{CM})$, Vietnamese $(\mathrm{V})$, and Korean $(\mathrm{K})$.

Trained bilingual, bicultural community health workers ([CHWs]; as partners in research,) representing the four language groups assisted investi- gators with translating materials, recruiting, and facilitating group discussions. A team approach was used to translate all study materials into English to assure linguistic validity. For this process, a multilingual research team was formed which included one of the principal investigators ([PI]; there were two PIs), a co-investigator, four bi-lingual or multi-lingual CHWs (who spoke English plus one or more other Asian-based languages), and a consultant. The team discussed and resolved ambiguities and arrived at an agreement for meaningful translation.

We recruited 55 older Asian immigrants from AHSC. This convenient and purposive sample included persons who self-identified as an immigrant to the United States, age 55 or older, having a primary language of Chinese (Cantonese or Mandarin), Vietnamese, or Korean. Language groups were organized based on constituents demographics. No Japanese-speaking persons enrolled in the study. The sample size was determined to be sufficient to achieve data saturation for intra- and inter-group comparison (Hennink, Kaiser \& Marconi, 2017). Grocery gift cards (\$10) were given as remuneration at the end of the group discussions.

\section{Data collection and analysis}

Scientific group-level assessment (GLA)

90-minute group discussions were held. In accordance with GLA methodology, data collection and initial analysis occurred concurrently in real-time with input from participants and facilitators. (We modeled our methods after Vaughn \& Lohmueller, 2014.) Six steps were followed: climate setting, generating data, appreciating perspective, reflecting, understanding the data, and selecting themes. GLA discussions provided timely and valid data.

In the climate setting step, facilitators sought to build trust with participants while considering cultural expectations for participating in research. Participants engaged in traditional social activities to create a comfortable atmosphere (a minimal dance exercise for Vietnamese, an armstretching activity for Koreans, and light general talk and laughter for Chinese-speaking persons). In the generating data step, investigators and $\mathrm{CHWs}$ introduced themselves and the research project. (CHWs were trained in advance using the Interactive CO-Learning for Research Engagement and Education [ICOREE] method; ${ }^{1}$ Nguyen-Truong \& ${ }^{1}$ Fritz et al., 2018). A scripted 12-minute talk was presented introducing smart homes alongside relevant pictures (e.g., showing installed sensors). Hand-held visuals of motion sensors were passed around. Then, CHWs facilitated participatory group discussions using a semi-structured, open-ended interview guide 
Table 1. Semi-Structured, open-ended interview guide.

Baseline Level of Comprehension of What a Health-Assistive Smart Home Monitoring Is

"Is there anyone that would be willing to describe back to me what the smart home is?"

[Or, "Could you please describe back to me your understanding of the smart home and what it can do?"]

Perceptions of Health-Assistive Smart Home Monitoring

"After seeing this [12 minute talk introducing the smart home including hand held visuals depicting sensors and data, and an actual sensor] what do you think?"

"What are the first thoughts that come to your mind?' [Or, "Please tell me the first thought that comes to your mind?"]

"What stood out to you?"

Feelings Regarding Health-Assistive Smart Home Monitoring

"As you think about sensors that potentially help monitor you when you are sick or ill, how does that feel?" [Or,

"As you think about sensors that potentially help monitor you as you grow older, how does that feel?"]

"Does the idea of having these sensors monitoring you make you feel..."

"Is it a good thing?" ["If so, what about the sensors feels like it is a good idea?"]

Barriers to Health-Assistive Smart Home Monitoring

"Is there anything about the idea of sensors that bothers you?" ["If it bothers you, what concerns do you have?"]

"What is the first thing that comes to your mind that is concerning?"

(Table 1). Participants were assigned to one of the four language groups. All four group discussions were held concurrently (start times were staggered) in different conference rooms of the community health center.

Two CHWs were assigned to each group. One CHW facilitated the discussion while the other took notes in participants' native language and simultaneously transcribed main points in English. The main points were written on large adhesive chart paper and verified with participants in real-time to assure the trustworthiness of the data (Lincoln \& Guba, 1985). In the appreciating perspective step, CHWs reviewed the main points and encouraged participants to add anything that came to mind. In the reflecting step, participants quietly thought about what the group's responses meant as a whole and added any summative thoughts. In understanding the data step, participants identified common themes from the content (i.e., written on the charts). In the selecting themes step, participants selected and prioritized common themes.

Trained observers took field notes to record intra-group dynamics. Investigators debriefed with CHWs and observers after participants left. Impressions of group processes and interactions were reviewed and investigators addressed questions and concerns (Table 1).

\section{Scientific conventional content analysis}

A data-analytic team was formed to further examine the themes generated by participants during group-level assessment (Hseih \& Shannon, 2005). This team included four individuals: two primary investigators (one non-Hispanic White; one bilingual/bicultural English-Vietnamese speaking); one English-speaking community consultant with a community health background in Asian American and Pacific Islander communities; and, finally, a Chinese-Vietnamese multi-lingual, mul- ti-cultural pharmacist with a community health background (who also took field notes).

Each analytic team member independently read the transcripts (written in English) from each of the four language groups. Each member then wrote a one-page simple summary of intra-group findings, which included a statement integrating Rogers' Diffusion of Innovation Theory, and shared it with the other team members. Each member also independently hand-coded the transcripts and sorted codes into potential inter-group themes. These were sent to the analytic team lead who compiled all team members' coded themes on an Excel $^{\circledR}$ spreadsheet and added supporting original text as well as the originating source. Then, all team members except the pharmacist met to discuss, clarify, and reach an agreement on intragroup findings. (The pharmacist joined again in the third round of analysis. See the section below on rigor.) The analytic team then reviewed the compiled information and reached an agreement on inter-group findings. The analytic team met three times to discuss emerging themes. During this process, each member documented their reflections and kept an audit trail. Codes were assigned representing each language-based group (i.e., CC, CM, V, and K). Common impressions informed intra-group and inter-group themes. Minor themes were subsumed by major themes. No themes were dropped. Analytic team consensus was sought and achieved.

\section{Rigor}

Culture and language concepts influenced concurrent data collection and analysis (CEnR), and analytic team findings. Team translation was used to enhance the trustworthiness of the data. A multicultural, multilingual analytic team approach accommodated for interpretation variances and enhanced credibility. Having the pharmacist join in the third round of analytic team discussions allowed the team to verify that final themes made practical sense and aligned with 
Table 2. Sociodemographic and background characteristics of the participants $(N=55)$.

\begin{tabular}{|c|c|c|}
\hline & Mean & $(S D)$ \\
\hline Age, years & 72.13 & $(6.03)$ \\
\hline Age at immigration, years & 47.25 & $(14.91)$ \\
\hline Years lived in the United States & 26.04 & $(13.23)$ \\
\hline Birth place & $n$ & $(\%)$ \\
\hline China & 28 & $(50.91)$ \\
\hline North Vietnam & 4 & $(7.27)$ \\
\hline Central Vietnam & 2 & $(3.64)$ \\
\hline South Vietnam ${ }^{\mathrm{a}}$ & 9 & $(16.36)$ \\
\hline South Korea & 11 & (20) \\
\hline Cambodia $^{b}$ & 1 & $(1.82)$ \\
\hline \multicolumn{3}{|l|}{ Gender } \\
\hline Female & 33 & $(60)$ \\
\hline Male & 22 & (40) \\
\hline \multicolumn{3}{|l|}{ Marital status } \\
\hline Single, have never been married & 2 & (3.64) \\
\hline Currently married & 36 & $(65.45)$ \\
\hline Separated & 2 & $(3.64)$ \\
\hline Divorced & 4 & $(7.27)$ \\
\hline Widowed & 11 & $(20)$ \\
\hline
\end{tabular}

\section{Education}

Elementary

Some middle school

6

Some high school

(10.91)

Some college

7

15

10

(12.73)

(27.27)

Graduated from college

17

(18.18)

(30.91)

Total household income before taxes

Less than $\$ 15,000$

38

$\$ 15,000-30,000$

13

$\$ 30,001-50,000$

$\$ 50,001-75,000$

$\$ 75,001-100,000$

Equal or more than $\$ 150,001$

How well do you speak English?

$\begin{array}{lcc}\text { Not at all } & 13 & (23.64) \\ \text { Not well } & 28 & (50.91) \\ \text { Well } & 12 & (21.82) \\ \text { Very well } & 2 & (3.64)\end{array}$

Note. $\mathrm{a}=$ One participant identified as Korean and was born in South Vietnam. $\mathrm{b}=$

One participant identified as Vietnamese and was born in Cambodia.

originally observed group discussions. Observers' field notes of group interactions provided an additional depth of understanding. Reflexivity was used throughout the analytic process as a technique to address the influence of personal biases on findings.

\section{Results \\ Sample description}

$(1.82)$

.

Thirty-three Asian immigrant women $(\mathrm{n}=33$, $60 \%$ ) and twenty-two men $(n=22,40 \%)$, aged 57 to 83 years, participated in the study. The mean age of participants was 72.13 years $(S D=6.03)$, and the mean years lived in the United States was 26.04 ( $\mathrm{SD}=13.23$ ). About 50.91 percent ( $\mathrm{n}$ $=28$ ) of participants reported not speaking English well. Sixteen (29.09\%) participants preferred Cantonese whereas $12(21.82 \%)$ preferred Mandarin, and 15 (27.27\%) and 12 (21.82\%) preferred Vietnamese and Korean respectively (Table 2 ).

Five clear inter-group (i.e., transcultural) main themes were identified across the four language groups: (1) Safety, (2) Features and Functionality, (3) Trusting the Technology, (4) Households and Family, and (5) Access - cost, internet, knowledge, and literacy. See Table 3 for details in the differences among the four language groups as intra-group themes.

\section{Safety}

The main idea regarded the capability of smart homes to assist with safety. Provision of safety by the smart home fell into two idea categories: assisting with a medical emergency and keeping safe from crime. Study participants in all four groups brought up the idea that having a smart home would provide increased safety in their living environments. For example, the Cantonese group questioned whether the smart home might ... "call 911 if there is a threat to life or property"? and the Mandarin group described it as "protecting my life if I have a sudden health emergency at home". Participants also perceived the smart home as a tool that might remind older adult immigrants to engage in healthy behaviors such as meditating, walking, or drinking tea. The Vietnamese group indicated the smart home would

All groups discussed the idea of being safe from crime. In response to the semi-structured question "I need to consider this before I move forward with having a smart home for health assistance..." one Korean participant responded with another question (a common cultural communication technique), "What if a thief steals a

Table 3. Intra-group themes from four language-based groups of older Asian immigrants and connection to major intergroup (transcultural) themes. Transcultural theme codes listed in far right column.

\begin{tabular}{|c|c|c|}
\hline Language group & Intra-group themes & Inter-group themes \\
\hline Korean & $\begin{array}{l}\text { limited technology knowledge }(A) \text {, functionality }(F) \text {, household } \\
\text { makeup }(H) \text {, privacy }(T) \text {, threats }(S, T) \text {, availability and cost }(A) \text {, } \\
\text { Asian culture }(H)\end{array}$ & \multirow{4}{*}{$\begin{array}{l}\text { (S) Safety } \\
\text { (F) Features and Functionality } \\
\text { (T) Trusting the Technology } \\
\text { (H) Households and Family } \\
\text { (A) Access: Cost, Internet, } \\
\text { Knowledge, Literacy }\end{array}$} \\
\hline Vietnamese & $\begin{array}{l}\text { limited technology knowledge }(A) \text {, smart home capacity }(\mathbf{F}) \text {, } \\
\text { availability }(A) \text {, accessibility }(A) \text {, safety }(S) \text {, cost }(A) \text {, Asian homes }(H)\end{array}$ & \\
\hline Chinese Cantonese & $\begin{array}{c}\text { access }(A), \text { radiation concerns }(T) \text {, user friendly }(F) \text {, installation }(A) \text {, } \\
\text { technology knowledge required }(A)\end{array}$ & \\
\hline Chinese Mandarin & $\begin{array}{c}\text { access }(A) \text {, technology knowledge required }(A) \text {, emergency concerns } \\
(S, T) \text {, user friendly }(F) \text {, locus of control }(A) \text {, language }(\mathbf{F}, \mathbf{A})\end{array}$ & \\
\hline
\end{tabular}


glance and breaks into your home?" Mandarinspeaking participants discussed the potential for assistance "... if someone robs my home...." To participants, the idea of being monitored by a home that could assess safety and health was appealing. However, questions arose regarding whether smart homes might also be an avenue for introducing crime. Multiple participants voiced concern about who would have access to the data and what would happen should there be a data breach. Provision against criminal activity was juxtaposed with the possibility that criminal access might be introduced.

Comfort emerged as a sub-theme to Safety. All groups agreed that it would be comforting to live in a smart home because they would feel safer. The word harm, an antonym to safety, was used by participants when expressing the need, and desire for, comfort and safety. Multiple participants expressed concern that if a smart home was "broken" it could cause "harm" (CM), as opposed to providing comfort. Participants were also concerned that their elders could develop an unhealthy dependence on a smart home (CM) leading to reduced social interactions (K, CC, and $\mathrm{V}$ participants) and a lack of suitable human response to changing health conditions.

\section{Features and functionality}

Many questions surfaced across all groups regarding how smart homes work. Questions about smart home features and functionality saturated the data early on across all transcripts. Many participants reported the main reason for enrolling in this study regarded a desire to learn about smart homes. Several astute questions were recorded, such as "... [Can] the sensor detect the senior sleeping while watching TV?" (K) and "... can [I] use this device in another country? Is it flexible with travel?" (K), and "How long will it last? Will it break easily?" (CM).

Timely nursing interventions were perceived as a desirable feature. One participant shared "It is no use if the sensor only detects [a change] ... The senior might die if [the] nurse intervenes too late" (K). There was interest in the smart home being able to move beyond simple monitoring; participants wanted it to interact with them. They also wanted this interaction to occur in their preferred language. Multiple participants asked whether a smart home would be able to "communicate with me in [my language]" (CC, CM, V, and $\mathrm{K}$ ). The idea of having to interact with technology using a second or third language (not native to their home country) appeared to be a daunting thought.

All groups were concerned about functionality and wanted to understand how the technology works. One participant stated, "There are many rooms in a house, how can the sensor monitor all of the rooms?" (V). Another group highlighted two concerns, stating, "II] hope the sensor will be able to identify residents and visitors" and that there would not be a "power outage or no battery" (CM). The Vietnamese group recognized that "The device only helps monitoring patients and [does] not cure patients". Additionally, participants indicated smart homes would need to be user friendly. For example, the Mandarin group indicated, "if the control interface is simple, language will not be a problem".

\section{Trusting the technology}

Trust was a salient idea identified in all transcripts. The idea of trust was connected to language about appropriate and accurate functioning. All groups questioned whether the smart home would work as intended; a key concept related to trust. Older immigrants indicated if a smart home did not function as intended, the trust would be broken and not likely re-established.

The Korean group discussed the smart home's potential to help their respected elders living in Korea. Participants indicated they did not want their elders to "die alone" and inferred a technology assisting with such an important task would be a trusted technology. However, both the Mandarin and Korean groups were concurrently concerned about technical failures, indicating "during an emergency, help won't come" (CM). Words like "emergency" and "over-reliance" on technology and "family" were connected in the transcripts. The implication was that technology failures would result in distrust; however, a family could be consistently trusted.

The Cantonese group included one divergent voice, a person who repeatedly spoke of radiation emitting from the sensors, indicating the potential for the "device [to] contain radiation?" The analytic team determined the concern for harm from radiation represented a lack of trust in the technology.

Cantonese, Mandarin, and Vietnamese groups (not Korean) hinted at privacy concerns, though privacy was not overtly discussed by them. The Korean group was the only group to spend time on the topic of privacy. Participants instead asked about data breaches and data accuracy. The specific concern was that data inaccuracies would lead to misconceptions about health needs.

\section{Households and family}

Connection with family was a common idea across all groups. Organic discussion of how a smart home would fit with family and the cultural emphasis on caring for, and connecting with, family and community elders were noted. All groups highlighted the necessity of remaining connected 
with humans when adding this technology to the home. It was suggested that smart homes should bring families together and assist children in caring for their elders. The Cantonese group pointed out that "less interaction with family/friends/neighbors" was not healthy or wise. These participants indicated that relying on humans to respond in a time of need can only occur when relationships are nurtured. However, they also indicated that nurturing relationships and using a smart home were not seen as mutually exclusive. The Korean group indicated "before [a] smart home is utilized, we need to build a network with [our] neighbors, such as church and family".

The idea that many immigrant families have multiple generations and several family members living in the same home surfaced across all transcripts. The Vietnamese group asked, "Is the smart home able to distinguish the older adult and the children or other people living under the same roof?" The Korean group indicated as long as smart homes can accommodate multiple people "there is no conflict with my culture".

\section{Access}

Participants wanted to know how they could obtain a smart home. Gaining access appeared to be important to their perceptions of future healthcare. Cost, internet, and knowledge emerged as potential barriers to access, should the older adult lack those same resources.

\section{Cost}

All groups indicated that smart homes must "cost a lot" (CC) or that it could be a "big burden for seniors [who are] low income" (K). Statements of "[I] don't have enough money" (V) and questions regarding "who will pay for them?" (CM) were common. One participant indicated a "senior discount" (K) would help and others verbalized their agreement. The Cantonese group summarized it best stating, "the toughest issue to address ... is [the] financial aspect".

\section{Internet}

A major perceived barrier to the adoption of the smart homes was not having internet. Statements were made such as "[the] senior living alone rarely has internet in his/her home" (K). Installing equipment and hooking it up to the internet without assistance was perceived as an additional barrier.

\section{Knowledge and literacy}

Participants expressed unsureness about learning to use smart home. A Mandarin participant indicated they would need the knowledge they currently did not have, "knowledge on how to use technology". A Korean participant indicated "It is very hard for me to adapt myself to the changes and use all the good devices and technology". However, despite thinking they lacked the necessary knowledge, a language in the transcripts appeared to indicate that participants across all groups (except the person concerned about radiation) were open to using a smart home. For example, a participant indicated "I am openminded to try new things" (CC).

The majority of participants were unfamiliar with the idea of smart homes but they appeared to quickly understand its features and functionality upon hearing about them. Understanding how to use a smart home was on the minds of participants, and their ability to learn this seemed connected to the capacity to use their native language while learning and using the smart home.

\section{Intra-group themes}

The Korean speaking group spent more time talking about privacy than the other groups. Thus, we identified it as an intra-group theme. One Korean participant indicated that "there should be privacy issue" [sic] while multiple other group participants indicated "there is no privacy issue".

The Vietnamese group spent more time ascertaining and discussing the smart home's capabilities than the other groups. Many more questions about the smart home's features and functions were asked by the Vietnamese group than by other groups. The Vietnamese group was particularly interested in the smart home's ability to differentiate between elders and children and grandchildren living in the same home. They also highlighted that monitoring does not equate to action, or "cure of the problem". They suggested the smart home should have familyfocused intervention capabilities.

The Chinese Cantonese group was uniquely interested in the installation process - who would do it and whether attaching the sensors might damage the walls and ceilings. They spent more time focusing on the pragmatic aspect of sensor maintenance (e.g., changing batteries). One participant repeatedly brought up the idea of harm from radiation. We identified installation and radiation as intra-group themes due to the time spent on these topics and their distinctiveness to this group.

The Chinese Mandarin group uniquely appeared to be more concerned with the decision-making process for 'allowing' smart home installation. Several participants indicated that they would need permission from others (e.g., a property owner, family member) before they would agree to smart home installation. This seemingly external locus of control regarding a decision to adopt was juxtaposed with a strong internal locus of control that regarded the smart home's features 
and functionality. For example, this group strongly indicated they wanted the smart home to communicate with them in their language (Mandarin). They also recognized the potential to become too reliant on the technology, thus potentially reducing the capacity for self-management of care.

\section{Discussion}

Investigators encountered great interest in the smart home's capabilities. These groups of diverse older Asian immigrants expressed a willingness to immediately try using a smart home. (Multiple participants approached investigators after the focus groups ended indicating they wanted to be put on a list to join a future study so they could obtain a smart home.) Our experience and findings lead us to believe that an overwhelming majority of these participants could be categorized as early adopters. This differs from the findings of Chung, Thompson, Joe, Hall, and Demiris (2016) who found that older Korean immigrants in the United States, as well as Korean citizens, were not immediately inclined to adopt a smart home because they viewed themselves as having good health. However, similar to Chung et al. (2016), we found that older adult immigrants wanted assistance with keeping safe from crime. Bicultural members of our analytic team shared that some immigrants, particularly from Vietnam, may be concerned with being victimized because of previous wartime experiences. Because of this, the analytic team concluded that smart homes should include culturally sensitive features designed to keep residents safe from crime.

The lack of privacy concern is a major difference between this study's findings and the findings of many other studies about older adults' perceptions of smart home monitoring. Jeong, Salvendy, and Proctor (2010) found privacy was less concerning to Koreans than Americans, however, privacy is highlighted in the extant gerontechnology literature as a major concern for most older adults. Multiple studies, including our own (Fritz et al., 2016), found that older adults expect privacy and protection from unwanted or unwarranted oversight (Austin, Dodge, Riley, Jacobs, Thielke, \& Kaye, 2016; Courtney, Demiris, Rantz, \& Skubic, 2008; Galambos et al., 2019; Galambos, Rantz, Back, Jun, Skubic, \& Miller, 2017). However, participants in those studies were primarily non-Hispanic Whites. Immigrant participants in this study did not highlight privacy as a concern (except for one Korean immigrant), rather, they focused on whether a smart home would function as intended, keep them safe from harm, and support family-based care and staying connected with others. Privacy was only briefly mentioned and was narrowly connected to concerns with criminally-driven data breaches.
The major concern with access to smart homes, including the associated costs, maybe uniquely tied to living in the United States. Citizens have to pay for much (or all) health-related care either at the time of service or through purchasing health insurance. Healthcare in the United States is not considered a basic human right, except in emergencies. Hence, the focus by these participants on access as it relates to the cost of equipment, monitoring services, and connectivity (i.e., internet costs) may have emerged as stronger themes than had the study been conducted in countries offering socialized models of care. Suggestions related to relying on family and community may be related to the realization that the cost of care in the United States can be quite high. Many older immigrants to the United States have few financial resources (Table 2). Additionally, we perceived that participants were guarding cultural traditions around family engagement and responsibilities.

This study uniquely highlights that a culturally sensitive approach to using smart homes is needed to facilitate human connection and prevent psychological harm. Many of the Vietnamese and Chinese-speaking participants in our study fled to the United States with their children and relatives during a war. Historical trauma and diaspora concerns appear to be ever-present in these persons ( ${ }^{1}$ Nguyen-Truong \& ${ }^{1}$ Fritz et al., 2018) and we encountered a stronger desire for family connectedness than appears to be indicated in previous smart home adoption studies, especially studies having a majority Caucasian, non-immigrant participant sample. Our findings suggest that older adult immigrants from diverse Asian traditions are willing to try using a smart home as long as it supports family connections and traditions. Smart homes were not perceived as an alternative to family-based care but rather something that could provide additional support to the whole family.

Bicultural members of the analytic team echoed concerns that smart homes may introduce loose filial piety (i.e., weakening of a child's duty to their parents) and the loss of tradition. These concerns were also reported by Chung et al. (2016) and Donovan \& Williams (2015) respectively. A new concern raised by our bicultural analytic team members regarded advanced care planning conversations between Asian elders and their children. Such conversations are taboo across multiple Asian cultures because the elder perceives it will bring bad luck and hasten death (Lee, Byon, Hinderer, \& Alexander, 2017). Discussions of smart home monitoring may lead to advanced care planning topics thereby upsetting the cultural tradition of not engaging in these types of conversations. Researchers conducting smart 
home studies should be aware of this dynamic and seek ways to support cultural traditions while introducing health-assistive technologies.

Rogers' diffusion of Innovation Theory offers two valuable insights related to how culture may influence adoption rates among older diverse Asian immigrants. First, the theory highlights the importance of peer-to-peer conversation within a social network. Second, the theory highlights the importance of understanding that different segments of populations have different needs and that an innovation's ability to meet specific needs is required for widespread adoption. Throughout our study, while planning, recruiting, and conducting the study, we encountered strong social networks and peer-to-peer connections. If Rogers' second theoretical insight concurrently existed (i.e., the smart home could meet specific needs) and the strong social network we encountered existed, the smart home would be well-positioned for widespread adoption among older diverse Asian immigrants.

\section{Limitations}

The main limitation of this study was introducing a smart home through pictures and conversation instead of having the participant actually live in a smart home. To truly understand their perceptions, participants would need to live in a smart home. Although real-time communityengaged participatory data analysis is a strength of this study and data saturation was reached for all identified themes, concurrently managing data collection, real-time analysis and translation may have resulted in minor data loss. The balance between participant burden and time for focus groups was weighed and 90 minutes was deemed appropriate. However, given more time other themes may have emerged. Other limitations include: some idea expressions could not be literally translated (but the meaning was achieved) and group facilitators (who were also translating) had only recently been introduced to the smart home and smart home research. Future research should focus on exploring the perceptions of other minority populations, including those defined by race, religion, gender, and sexual orientation who are living in a smart home.

\section{Implications}

Smart homes may assist with improving health and extending independence in older diverse Asian immigrants. Mobilizing community leaders could lead to culturally safe diffusion of this innovation within minority communities. Findings from this study could impact the design of smart home features. A desirable outcome for smart homes would be the inclusion of culturally sensitive features. Additionally, including immigrant persons in research studies is important for generating data representing diverse human experiences.

\section{Conclusions}

Smart homes that are capable of assisting older adults with aging-in-place are under development. The adoption of smart home monitoring by minority populations is not well understood. In this study, we explored the influence of culture on smart home adoption and we significantly increased sample size and diversity over prior studies on culture and adoption of smart home technology. We engaged older immigrants from diverse Asian backgrounds using a CEnR approach and found that these participants from four language groups (Korean, Vietnamese, Chinese Cantonese or Mandarin) were open to the idea of sensorbased monitoring for health-assistance. We conclude that: a) culture plays a role in whether older adult diverse Asian immigrants would use a smart home for aging-in-place, b) smart homes should be designed to primarily assist with keeping older adult immigrants healthy and safe from crime, c) smart homes must work as intended, and d) smart homes should include features and functions that accommodate, not negate, culturally influenced family-based activities in the home.

\section{Acknowledgements}

This research was supported in part by the Beta Psi Chapter of Sigma Theta Tau International Nursing Research Award and Washington State University Vancouver Research Mini-Grant. The authors thank Asian Health \& Service Center Executive Director Holden Leung, MSW and Drs. Catherine Van Son, PhD, RN, ANEF, Julie Postma, PhD, RN, Renee Hoeksel, PhD, RN, ANEF, at Washington State University College of Nursing. The authors thank all peer reviewers.

Portions of this work were presented at the Western Institute of Nursing's 2019 Research Conference in San Diego, California, USA.

\section{References}

Alberdi, A., Weakley, A., Goenaga, M., Schmitter-Edge- combe, \& Cook, D. J. (2018). Automatic assessment of functional health decline in older adults based on smart home data. Journal of Biomedical Informatics, 81, 119-130. https://doi.org/10.1016/j. jbi.2018.03.009

American Academy of Nursing. (2011). Code of Ethics. https://www.nursingworld.org/practice-policy/nursing-excellence/ethics/code-of-ethics-for-nurses/

Austin, J., Dodge, H., Riley, T., Jacobs, P., Thielke, S., \& Kaye, J. (2016). A smart-home system to unobtrusively and continuously assess loneliness in older adults. IEEE Journal of Translational Engineering in Health and Medicine, 4, 1-11. https://doi. org/10.1109/JTEHM.2016.2579638

Chen, K., \& Chan, A. H. S. (2014). Gerontechnology acceptance by elderly Hong Kong Chinese: A senior 
technology acceptance model (STAM). Ergonomics, 57(5), 635-52. https://doi.org/10.1080/0014013 9.2014.895855

Chung, J., Thompson, H. J., Joe, J., Hall, A., \& Demiris, G. (2017). Examining Korean and Korean American older adults' perceived acceptability of home-based monitoring technologies in the context of culture. Informatics for Health and Social Care, 42(1), 61-76. https://doi.org/10.3109/17538157.2016.1160244

Courtney, K. L., Demiris, G., \& Hensel, B. K. (2007). Obtrusiveness of information-based assistive technologies as perceived by older adults in residential care facilities: A secondary analysis. Medical Informatics and the Internet in Medicine, 32(3), 241-9. https://doi.org/10.1080/14639230701447735

Courtney, K., Demiris, G., Rantz, M., \& Skubic, M. (2008). Needing smart home technologies: The perspectives of older adults in continuing care retirement communities. Informatics in Primary Care, 16(3), 195-201.

Donovan, R., \&Williams, A. M. (2015). Care-giving as a Canadian-Vietnamese tradition: 'It's like eating, you just do it'. Health and Social Care in the Community, 23(1), 79-87. https://doi.org/10.1111/hsc.12126

Dzissah, D. A., Lee, J. S., Suzuki, H., Nakamura, M., \& Obi, T. (2019). Privacy enhanced healthcare information sharing system for home-based care environments. Healthcare Informatics Research, 25(2), 106-114. https://doi.org/10.4258/ hir.2019.25.2.106.

Fritz, R., Corbett, C., Vandermause, R., \& Cook, D. (2016). The influence of culture on older adults' adoption of smart home monitoring. Gerontechnology, 14(3), 146-156.

Galambos, C.M., Rantz, M., Craver, A., Bongiorno, M., Pelts, M., Holik, A., \& Jun, J.S. (2019). Living with intelligent sensors: Older adult and family perceptions. Computers, Informatics, \& Nursing, 37(11), 245-257. https://doi.org/10.1097/ CIN.0000000000000555

Galambos, C., Rantz, M., Back; J., Jun, J.S., Skubic, M., \& Miller, S., (2017). Older adults' perceptions and preferences of a fall risk assessment system: Exploring stages of acceptance model. Computers, Informatics, \& Nursing, 35(7), 331-337.

Helal, S., \& Bull, C. N. (2019). From smart homes to smart-ready homes and communities. Dementia and Geriatric Cognitive Disorders, 47, 157-163. https://doi.org/10.1159/000497803

Hennink, M. M., Kaiser, B. N., \& Marconi, V. C. (2017). Code saturation versus meaning saturation: How many interviews are enough? Qualitative Health Research, 27(4), 591-608. https://doi. org/10.1177/1049732316665344

Hong, Y. A., Zhou, Z., Fang, Y. (2017). Digital divide and health disparities in China: Data from a national longitudinal survey of CHARLS. International Medical Informatics Association and IOA Press, 245, 1301-1301. https://doi.org/10.3233/9781-61499-830-3-1301

Hseih, H. F., \& Shannon, S. E. (2005). Three approaches to qualitative content analysis. Qualitative Research, 15(9), 1277-1288. https://doi. org/10.1177/1049732305276687
Hunter I., Elers, P., Lockhart, C., Whiddett, D., Guesgen, H., \& Singh, A. (2019). Technology to assist aging in place: The perspective of health organizations. Studies in Health Technology and Informatics, 264, 1688-1689. https://doi.org/10.3233/SHTI190598

International Federation on Ageing. (2011). Ageing in place: GLOBAL. Global Ageing: Issues \& Actions, 11(2), 1-53. Toronto, Canada.

Jeong, K. A., Salvendy, G., \& Proctor, R. W. (2010). Smart home design and operation preferences of Americans and Koreans. Ergonomics, 53(5), 636-660.

Lee, M., Byon, H., Hinderer, K., \& Alexander, C. (2017). Beliefs in advance care planning among Chinese Americans: Similarities and differences between the younger and older generations. Asian/Pacific Island Nursing Journal, 2(3), 83-90. https://doi. org/10.9741/23736658.1055

Lincoln, Y. S., \& Guba, E. G. (1985). Establishing trustworthiness. In Naturalistic inquiry (pp. 289-331). Beverly Hills, CA: Sage.

Lundin, M., \& Mäkitalo, A. (2017). Co-designing technologies in the context of hypertension care: Negotiating participation and technology use in design meetings. Informatics for Health and Social Care, 42(1), 18-31. https://doi.org/10.3109/17538157.2015.1113176

Mara, B., Gill, G. K., Babacan, H., \& Donahoo, D. (2011). Digital technology, diabetes and culturally and linguistically diverse communities: A case study with elderly women from the Vietnamese community. Health Education Journal, 71(4), 491504. https://doi.org/10.1177/0017896911407054

National Institute on Aging. (2017). Aging in place: Growing old at home. Retrieved from https://www.nia.nih. gov/health/aging-place-growing-old-home

${ }^{1}$ Nguyen-Truong, C. K. Y., 'Fritz, R. L., Lee, J., Lau, C., Le, C., Kim, J.,Van Son C. (2018). Interactive colearning for research engagement and education (I-COREE) to build capacity between community partners and academic researchers. Asian/Pacific Island Nursing Journal, 3(4), 126-138. https://doi. org/10.31372/20180304.1030. [ ${ }^{1}$ Co-first authors]

Nguyen-Truong, C. K. Y., Pedhiwala, N., Nguyen, V., Le, C., Le, T. V., Lau, C., Lee, J., \& Lee-Lin, F. (2017). Feasibility of a multi-component breast health education intervention for Vietnamese American immigrant women. Oncology Nursing Forum, 44(5), 615-625. https://doi.org/10.1188/17.ONF.615-625.

Peek, S. T. M., Luijkx, K., Rijnaard, M. D., Nieboer, M. E., vanderVoort, C. S., Aarts, S., Wouters, E. (2016). Older adults' reasons for using technology while aging in place. Gerontology, 12(5), 1689-1699. https://doi.org/10.1159/000430949

Rantz, M. J., Skubic, M., Popescu, M., Galambos, C., Koopman, R. J., Alexander, G. L., Miller, S. J. (2015). A new paradigm of technology-enabled "vital signs" for early detection of health change for older adults. Gerontology, 61(3), 281-290. https:// doi.org/10.1159/000366518

Rogers, E.M. (1976). New product adoption and confusion. Journal of Consumer Research, 2, 290-301.

Rogers, E.M. (2003). Diffusion of innovations (5th ed.). New York: Free Press

Rogers, E.M. (2004). A prospective and retrospective look at the diffusion model. Journal of Health Com- 


\section{Smart home adoption perceptions}

munication, 9, 13-19.

Sahin, I. (2006). Detailed review of rogers' diffusion of innovation theory and educational technology-related study based on rogers' theory. The Turkish Online Journal of Educational Technology, 5(2), 14-23.

Sprint, G., Cook, D. J., Fritz, R., \& Schmitter-Edgecombe, M. (2016). Using smart homes to detect and analyze health events. Computer, 49(11), 29-37. https://doi.org/10.1109/MC.2016.338

Vaughn, L. M., \& Lohmueller, M. A. (2014). Calling all stakeholders: Group level assessment (GLA) - a qualitative and participatory method for large groups. A Journal of Applied Social Research, 38(4), 336-355. https://doi.org/10.1177/0193841X14544903

World Health Organization. (2018). Ageing and health. Retrieved from https://www.who.int/news-room/ fact-sheets/detail/ageing-and-health

Ye, J., Stevenson, G., \& Dobson, S. (2016). Detecting abnormal events on binary sensors in smart home environments. Pervasive and Mobile Computing, 33, 32-49. 